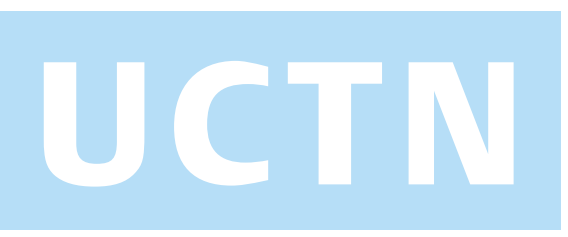

A 42-year-old man presented with a 2year history of vague epigastric discomfort, intermittent nausea and vomiting. The patient denied weight loss. Results of the laboratory studies were normal except for a persistently elevated lipase level within a range of $150-300 \mathrm{U} / \mathrm{l}$ (normal range, $17-55)$. The serum albumin level was $3.8 \mathrm{~g} / \mathrm{dl}$ (normal range, 3.4-4.7) and the CA $19-9$ was $4 \mathrm{U} / \mathrm{ml}$ (normal range, $0-37)$. A high resolution computed tomography (CT) scan of the abdomen (Figure $\mathbf{1 A}$ ) revealed a circumferential intramural cystic lesion (arrow) extending from the descending duodenum to the transverse duodenum. The cystic lesion measured $5.5 \times 5 \mathrm{~cm}$ at the level of the pancreatic head and significantly constricted the lumen of the second and third portion of the duodenum. A coronal reconstruction of the axial CT images

\section{Duodenal Duplication Cyst}

provided a better delineation of a complex multilobular circumferential cyst (Figure 1B). An endoscopic retrograde cholangiopancreatography (ERCP) was attempted but cannulation of the ampulla failed due to luminal narrowing. Upper endoscopy demonstrated a narrowed duodenal lumen and a lumpy mucosal surface with patchy erythema (Figure 2). Endoscopic ultrasonography (EUS) examination of the duodenum was difficult, but confirmed a large cystic lesion in the duodenum. Because of the progressively obstructing symptoms and the inability to completely exclude a cystic tumor, the patient chose to undergo surgical resection. Intraoperatively, a cystic mass, approximately $11 \mathrm{~cm}$ in length, was found. It appeared to involve the head of the pancreas, and the second, third, and fourth portions of the duodenum (Figure 3 ). Be-

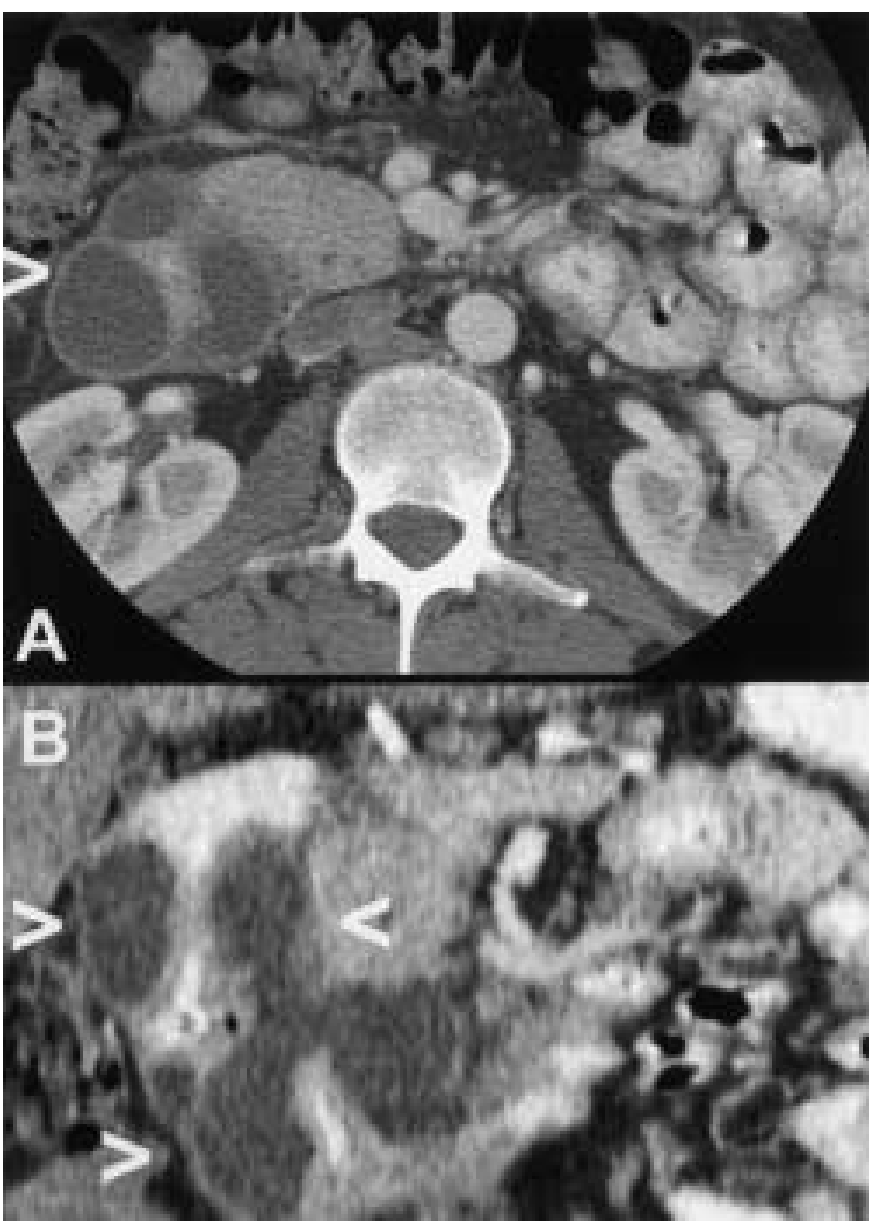

Figure 1 A A highresolution computed tomography (CT) image of the abdomen reveals a circumferential cystic lesion (arrow) extending from the descending duodenum to the transverse duodenum. B A three-dimensional reconstruction of the $\mathrm{CT}$ image delineates the complex multilobular cystic lesion. cause of the uncertain nature of the lesion, as well as its intimate involvement with the head of the pancreas, a pyloruspreserving pancreatoduodenectomy was performed. Reconstruction included an end-to-side pancreaticojejunostomy, a hepaticojejunostomy and a retrocolic duodenojejunostomy.

Microscopic examination of the cystic mucosa revealed cuboidal cell epithe-

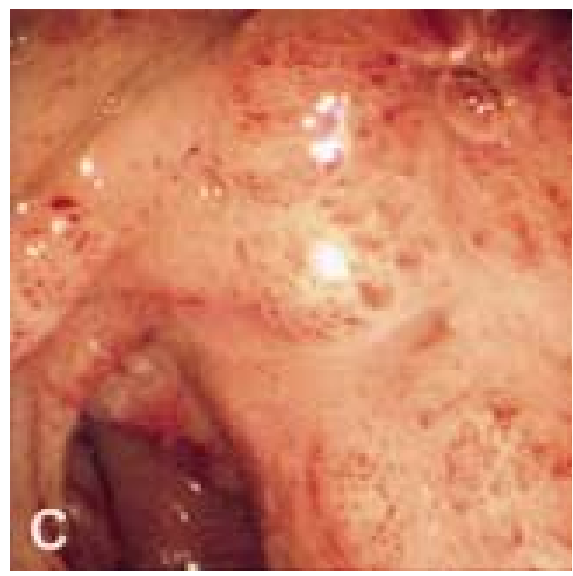

Figure 2 Endoscopic view demonstrates a narrowed duodenal lumen and a lumpy mucosal surface with patchy erythema.

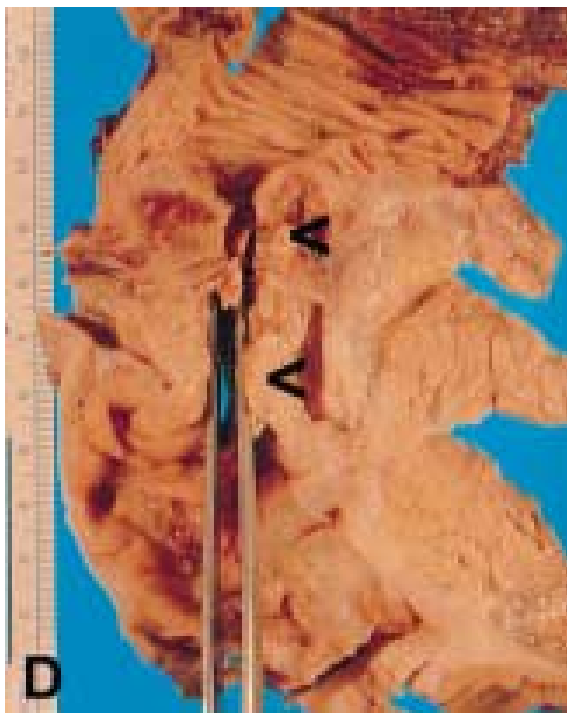

Figure 3 The resected specimen shows the multilobular cystic mass, approximately $11 \mathrm{~cm}$ in length. The arrows indicate two duodenal strictures. 
lium. Examination of the two strictured areas demonstrated dense fibrosis in the mucosa and submucosa of the cystic wall. The final pathological diagnosis was duodenal duplication cyst with two duodenal strictures (Figure 3, arrows).

Duodenal duplication cysts are rare and account for $5 \%$ of all enteric duplications. They are usually found in the medial wall of the first and second portion of the duodenum. Duodenal duplication cyst can present with gastric outlet or duodenal obstruction, recurrent pancreatitis and gastrointestinal hemorrhage [1,2]. Although CT scan, magnetic resonance cholangiopancreatography (MRCP), esophagogastroduodenoscopy (EGD) and EUS can suggest the diagnosis, this is usually confirmed at laparotomy. The treatment of duplication cysts is surgical resection or surgical cystoduodenostomy if the cyst is obstructing and symptomatic. Endoscopic drainage or removal of duodenal duplication cyst has been reported [3-5]. If the cyst involves the pancreatic head and biliary tract, the treatment of choice is pancreaticoduodenectomy [2]. The complex nature of the cystic lesion in our patient precluded endoscopic intervention and treatment.

\section{S.-J. Tang1, S. Raman 2, H. A. Reber ${ }^{3}$, R. Bedford 1, B. E. Roth ${ }^{1}$}

${ }^{1}$ Division of Digestive Diseases, Department of Medicine, Center for Health Sciences, UCLA School of Medicine, Los Angeles, California, USA

${ }^{2}$ Department of Radiology, Center for Health Sciences, UCLA School of Medicine, Los Angeles, California, USA

${ }^{3}$ Department of Surgery, Center for Health Sciences, UCLA School of Medicine, Los Angeles, California, USA

\section{References}

${ }^{1}$ Leffall, Jr LS, Jackson M, Press H, Syphax B. Duplication cyst of the duodenum. Arch Surg 1967; 94: 30-34

2 Rutledge PL, Warshaw AL. Persistent acute pancreatitis. A variant treated by pancreatoduodenectomy. Arch Surg 1988; 123: $597-600$
${ }^{3}$ Bulajic M, Savic-Perisic M, Korneti V et al. Use of endoscopy to diagnose symptomatic duodenal duplication cyst in an adult. Endoscopy 1991; 23: 234-236

${ }^{4}$ Dave P, Romeu J, Clary S et al. Endoscopic removal of an obstructing duodenal duplication cyst. Endoscopy 1984; 16: $75-76$

${ }^{5}$ Johanson JF, Geenen JE, Hogan WJ, Huibregtse K. Endoscopic therapy of a duodenal duplication cyst. Gastrointest Endosc 1992; 38: 60-64

\section{Corresponding Author}

\section{S.-J. Tang}

Center for Therapeutic Endoscopy and Endoscopic Oncology 16-130 Victoria Wing

St. Michael's Hospital

30 Bond Street

Toronto, Ontario

M5B IW8

Canada

Fax: $\quad+1-416-864-5449$

E-mail: tangs@smh.toronto.on.ca 